\title{
Fumonisins affect the intestinal microbial homeostasis in broiler chickens, predisposing to necrotic enteritis
}

\author{
Gunther Antonissen ${ }^{1,2^{*}}$, Siska Croubels ${ }^{2}$, Frank Pasmans ${ }^{1}$, Richard Ducatelle ${ }^{1}$, Venessa Eeckhaut ${ }^{1}$, \\ Mathias Devreese ${ }^{2}$, Marc Verlinden ${ }^{1}$, Freddy Haesebrouck ${ }^{1}$, Mia Eeckhout ${ }^{3}$, Sarah De Saeger ${ }^{4}$, Birgit Antlinger ${ }^{5}$, \\ Barbara Novak ${ }^{5}$, An Martel $^{1}$ and Filip Van Immerseel ${ }^{1}$
}

\begin{abstract}
Fumonisins (FBs) are mycotoxins produced by Fusarium fungi. This study aimed to investigate the effect of these feed contaminants on the intestinal morphology and microbiota composition, and to evaluate whether FBs predispose broilers to necrotic enteritis. One-day-old broiler chicks were divided into a group fed a control diet, and a group fed a FBs contaminated diet (18.6 mg FB1+FB2/kg feed). A significant increase in the plasma sphinganine/sphingosine ratio in the FBs-treated group $(0.21 \pm 0.016)$ compared to the control $(0.14 \pm 0.014)$ indicated disturbance of the sphingolipid biosynthesis. Furthermore, villus height and crypt depth of the ileum was significantly reduced by FBs. Denaturing gradient gel electrophoresis showed a shift in the microbiota composition in the ileum in the FBs group compared to the control. A reduced presence of low-GC containing operational taxonomic units in ileal digesta of birds exposed to FBs was demonstrated, and identified as a reduced abundance of Candidatus Savagella and Lactobaccilus spp. Quantification of total Clostridium perfringens in these ileal samples, previous to experimental infection, using cpa gene (alpha toxin) quantification by qPCR showed an increase in C. perfringens in chickens fed a FBs contaminated diet compared to control (7.5 \pm 0.30 versus $6.3 \pm 0.24 \log 10$ copies/g intestinal content). After C. perfringens challenge, a higher percentage of birds developed subclinical necrotic enteritis in the group fed a FBs contaminated diet as compared to the control (44.9 $\pm 2.22 \%$ versus $29.8 \pm 5.46 \%)$.
\end{abstract}

\section{Introduction}

Mycotoxins are naturally occurring secondary fungal metabolites produced both pre- and post-harvest in crops and other feed and food commodities. Fusarium, Aspergillus, and Penicillium are the most abundant mycotoxin producing mould genera contaminating feed and feed raw materials [1]. Fumonisins (FBs) are produced by Fusarium verticillioides, $F$. proliferatum, and other Fusarium species and are among the most widespread mycotoxins [2]. FBs are ubiquitous contaminants of corn and other grain products [3]. A global survey on

\footnotetext{
* Correspondence: Gunther.Antonissen@UGent.be

'Department of Pathology, Bacteriology and Avian Diseases, Faculty of Veterinary Medicine, Ghent University, Salisburylaan 133, 9820 Merelbeke, Belgium

${ }^{2}$ Department of Pharmacology, Toxicology and Biochemistry, Faculty of Veterinary Medicine, Ghent University, Salisburylaan 133, 9820 Merelbeke, Belgium

Full list of author information is available at the end of the article
}

the occurrence and contamination levels of mycotoxins in feed raw materials and finished feed for livestock animals showed that $54 \%$ of 11439 tested samples were contaminated with FBs [4]. FBs were most frequently detected in South American (77\%), African (72\%) and Southern European (70\%) samples, and less frequently in Oceania (10\%) [4]. The economic impact of in animal feed is rather difficult to measure because information about subclinical effects on animal health and productivity losses due to chronic low level exposure is limited. $\mathrm{Wu}$ [5] estimated the annual economic losses in the USA due to FBs in animal feed to be US\$ 1-20 million and US\$ 30-46 million, in a normal and an outbreak year of Fusarium ear rot, respectively.

More than 28 FB homologues have been identified. Fumonisin $\mathrm{B}_{1}\left(\mathrm{FB}_{1}\right)$ is the most common and the most thoroughly investigated mycotoxin because of its toxicological importance. $\mathrm{FB}_{2}$ and $\mathrm{FB}_{3}$ are less prevalent, and 
differ structurally from $\mathrm{FB}_{1}$ in the number and position of hydroxyl groups [2]. FBs competitively inhibit the ceramide synthase and, as a result, interfere with the biosynthesis of ceramides and sphingolipids of cell membranes $[2,6]$. Clinical outbreaks have been reported in horses (equine leucoencephalomalacie, ELEM) and pigs (porcine pulmonary edema, PPE). These animal species are regarded as the most susceptible to the effects of FBs [2]. In general, poultry are considered to be quite resistant toward the deleterious effects of FBs. Also species differences occur, laying hens and broilers are less sensitive to FBs compared to turkeys and ducks [7-11]. In broilers, systemic uptake of $\mathrm{FB}_{1}$ after oral exposure is low, indicating that the absorption is negligible [12]. Following the consumption of FBs contaminated feed, the intestine is the first organ to be exposed to these toxins and negative effects on intestinal tissues have been reported [13]. The jejunum of broilers exposed to high $\mathrm{FB}_{1}$ concentrations ( $\geq 100 \mathrm{mg} / \mathrm{kg}$ feed) for 28 days displays a reduced villus height $(\mathrm{VH})$ and villus-to-crypt ratio (V:C) [14]. Besides a mild villus atrophy, also goblet cell hyperplasia is observed in broiler chicks exposed to high levels of $\mathrm{FB}_{1}(300 \mathrm{mg} / \mathrm{kg}$ feed) for 2 weeks [15]. It has been shown in vitro that $\mathrm{FB}_{1}$ has a toxic effect on both undifferentiated and differentiated porcine intestinal epithelial cells (IPEC-1). The effect of $\mathrm{FB}_{1}$ on epithelial cell proliferation correlates with a cell cycle arrest in the G0/G1 phase [16]. A negative effect of $\mathrm{FB}_{1}$ on the expression of cell junction proteins $\mathrm{E}$-cadherin and occludin, and consequently on the intestinal epithelial integrity, has been shown in vivo in pigs $[17,18]$. Furthermore, $\mathrm{FB}_{1}$ modulates intestinal immunity by decreasing the expression of several cytokines, for example interleukin (IL)-1 $\beta$, IL-2, IL-8, IL-12p40 and interferon (IFN) $-\gamma$ in pigs [19-21]. It has been shown that exposure of pigs to $0.5 \mathrm{mg} \mathrm{FB} / 1 \mathrm{~kg}$ bodyweight (BW) for 6 days enhanced intestinal colonization and translocation of a septicemic Escherichia coli (SEPEC) [20]. Feeding a FBs contaminated diet (11.8 mg $\mathrm{FB}_{1}+\mathrm{FB}_{2} / \mathrm{kg}$ feed) for 9 weeks transiently modified the faecal microbiota composition in pigs. Co-exposure to FBs and Salmonella Typhimurium amplified this phenomenon [22]. At present it is unclear what may be the consequences of long term exposure to low levels of FBs.

In poultry, necrotic enteritis (NE) is caused by netB producing Clostridium perfringens strains. C. perfringens is a Gram-positive spore-forming anaerobic bacterium that is commonly found in the environment and the gastro-intestinal tract of animals and humans as a member of the normal microbiota [23-25]. NE in chickens is still an important intestinal disease despite the application of preventive and control methods, including coccidiosis control. The acute form of the disease causes mortality without premonitory symptoms. The more frequently occurring subclinical form is characterized by intestinal mucosal damage without clinical signs or mortality, leading to decreased performance $[24,26]$. However, healthy birds often carry netB positive C. perfringens without showing any clinical symptoms of $\mathrm{NE}$ [24]. An outbreak of NE is a complex process requiring one or a number of predisposing factors rather than just the presence of pathogenic C. perfringens [27-29]. Preexisting mucosal damage caused by coccidiosis, high protein feed (including fishmeal) and indigestible nonstarch polysaccharides are well known predisposing factors [29]. Recently, it was shown that the mycotoxin deoxynivalenol (DON) is also a predisposing factor for the development of NE, through damage to the epithelial barrier and an increased intestinal nutrient availability for clostridial proliferation [30]. Although FBs are ubiquitous contaminants in poultry feed, information about their impact on the intestinal microbial homeostasis in broiler chickens is lacking.

The objective of this study was to evaluate the effect of FBs on the intestinal microbial homeostasis, at concentrations approaching the European Union maximum guidance levels (20 mg FB $1+\mathrm{FB}_{2} / \mathrm{kg}$ feed) [31]. Therefore, the influence of $\mathrm{FBs}$ on the intestinal microbiota composition and intestinal morphology was investigated. In addition, an attempt was made to demonstrate the consequences of the effect of FBs on the intestinal microbial homeostasis in a subclinical necrotic enteritis model.

\section{Material and methods Fumonisins}

FBs (8.64mg $\mathrm{FB}_{1}+\mathrm{FB}_{2} / \mathrm{g}$ culture material) (Biopure Romer Labs Diagnostic GmbH, Tulln, Austria) were produced in vitro from a culture of $F$. verticillioides, and subsequently crystallized [32]. For the in vitro assessment of the impact of $\mathrm{FB}_{1}$ on growth and toxin production characteristics of $C$. perfringens, serial dilutions were prepared in tryptone glucose yeast (TGY) broth medium of a $\mathrm{FB}_{1}$ stock solution of $5000 \mu \mathrm{g} / \mathrm{mL}$ (Fermentek, Jerusalem, Israel) that had been prepared in anhydrous methanol and stored at $-20^{\circ} \mathrm{C}$.

\section{Bacterial strain and growth conditions}

C. perfringens strain 56 , a net $B^{+}$type A strain, was originally isolated from a broiler chicken with $\mathrm{NE}$ and has been shown to be virulent in an in vivo infection model $[30,33]$. The inoculum for the oral infection of chickens and in vitro experiments was prepared by culturing $C$. perfringens anaerobically overnight at $37{ }^{\circ} \mathrm{C}$ in brain heart infusion broth (BHI, Bio-Rad, Marnes-la-Coquette, France) or tryptone glucose yeast (TGY) broth medium, respectively. The colony forming units of C. perfringens $/ \mathrm{mL}$ was assessed by plating tenfold dilutions on Colombia 
agar (Oxoid, Basingstoke, UK) with 5\% sheep blood, followed by anaerobic overnight incubation at $37^{\circ} \mathrm{C}$.

\section{Animal experiment \\ Birds and housing}

The animal experiment was performed using nonvaccinated Ross 308 broiler chickens, obtained as one-dayold chicks from a commercial hatchery (Vervaeke-Belavi, Tielt, Belgium). Animals of both treatment groups, control diet and FBs contaminated diet, were housed in the same temperature controlled room, in pens of $1.44 \mathrm{~m}^{2}$, on wood shavings. Each group consisted of three pens of 34 birds, with approximately equal numbers of males and females. Animal units were separated by solid walls to prevent direct contact between animals from different pens. All cages were decontaminated with peracetic acid and hydrogen peroxide (Metatectyl $\mathrm{HQ}$, Metatecta, Kontich, Belgium) and a commercial anticoccidial disinfectant (Bi-OO-Cyst Coccidial Disinfectant, Biolink, York, UK) prior to the housing of the chickens. Water and feed was provided ad libitum. Chickens were not fasted before euthanasia. The animal experiment was approved by the Ethical Committee of the Faculty of Veterinary Medicine and Bioscience Engineering, Ghent University (EC 2012/194).

\section{Feed preparation and experimental diets}

All chickens were fed a starter diet during the first eight days of the trial, and subsequently a grower diet. The diet was wheat and rye based, with soybean meal as main protein source during the first 16 days. From day 17 onwards, the same grower diet was fed with the exception that fishmeal replaced soybean meal as main protein source $[30,33]$.

FBs contaminated feed was produced by adding lyophilized FBs culture material to a control diet. Mycotoxin contamination of both the control and FBs contaminated diet was analyzed by a validated multi-mycotoxin liquid chromatography-tandem mass spectrometry method (LCMS/MS) [34]. Three different batches of FBs contaminated feed were produced: a starter diet, a grower diet with soybean meal and a grower diet with fishmeal, respectively. Therefore, FBs culture material was added to $500 \mathrm{~g}$ of the corresponding batches of control diet. For each batch, this premix was then mixed with $5 \mathrm{~kg}$ of control feed to assure homogeneous distribution of the toxin and finally mixed for $20 \mathrm{~min}$ in the total amount of feed needed for each batch. To test mycotoxin contamination, samples were taken at three different locations in each batch, subsequently pooled per batch and analyzed for mycotoxin contamination as described above. Trace amounts of nivalenol and DON were detected in the control feed $(0.059-0.116$ and $0.113-0.170 \mathrm{mg} / \mathrm{kg}$ feed, respectively). Analyzed mycotoxins, their limit of detection and limit of quantification were as previously described
$[30,34]$. The levels of FBs and all other tested mycotoxins in the different batches of control feed were below the limit of detection. The average levels of $\mathrm{FB}_{1}, \mathrm{FB}_{2}$ and $\mathrm{FB}_{3}$ in the different batches of FBs contaminated feed were $10.4 \mathrm{mg} / \mathrm{kg}, 8.2 \mathrm{mg} / \mathrm{kg}$ and $2.0 \mathrm{mg} / \mathrm{kg}$, respectively (Table 1). The average sum of FB1+FB2, $18.6 \mathrm{mg} / \mathrm{kg}$ feed was approaching the EU maximum guidance level in feed for poultry of $20 \mathrm{mg} \mathrm{FB} 1+\mathrm{FB} 2 / \mathrm{kg}$ (2006/576/EC) [31].

\section{Evaluation of the impact of FBs on broiler health}

The BW of all chickens was measured on day 1 and day 8. On day 15 , six birds $\left(3{ }^{\lambda} / 3\right.$ 우 $)$ per pen were euthanized using an overdose of sodium pentobarbital (Natrium Pentobarbital 20\%, Kela Veterinaria, Sint- Niklaas, Belgium). A blood sample was collected and subsequently a necropsy was performed. Blood samples were centrifuged $\left(2851 \times g, 10 \mathrm{~min}, 4{ }^{\circ} \mathrm{C}\right)$ and plasma was stored at $\leq-20{ }^{\circ} \mathrm{C}$ until sphinganine (Sa) and sphingosine (So) concentrations were analyzed. Sa/So is suggested to be the most sensitive biomarker to FBs intoxication in many animals [2]. Plasma Sa and So concentrations were analyzed by a commercial service provider (Biocrates Life Sciences AG, Innsbruck, Austria). Briefly, Sa and So were extracted from plasma and measured in the presence of internal standards using LC-MS/MS with electrospray ionization.

The BW and weight of different organs (liver, spleen, kidneys, proventriculus, ventriculus, bursa of Fabricius, heart and lungs) were recorded. The weight of each organ was converted to a relative percentage of the BW.

\section{Evaluation of the impact of FBs on intestinal morphology}

After measuring the length of the different small intestinal segments, $1 \mathrm{~cm}$ samples from the mid-duodenum, mid-jejunum and mid-ileum were collected and fixed in neutral-buffered formalin. Small intestinal segments were defined as duodenum encompassing the duodenal loop, jejunum between the end of the duodenal loop and Meckels diverticulum and ileum between Meckels diverticulum and the ileo-cecal junction. Villus height and crypt depth of mid-duodenum, mid-jejunum and mid-ileum were measured on hematoxylin and eosin stained histological paraffin sections using light microscopy with Leica LAS software (Leica Microsystems,

Table 1 Concentration of $\mathrm{FB}_{1}, \mathrm{FB}_{2}$ and $\mathrm{FB}_{3}$ in different batches of FBs contaminated diet

\begin{tabular}{|c|c|c|c|c|c|}
\hline Type of feed & Feeding period & $\mathrm{FB}_{1}$ & $\begin{array}{l}\mathrm{FB}_{2} \\
\mathrm{mg} /\end{array}$ & $\begin{array}{l}\mathrm{FB}_{1}+\mathrm{FB}_{2} \\
\mathrm{~kg} \text { feed }\end{array}$ & $\mathrm{FB}_{3}$ \\
\hline Starter & day $1-8$ & 8.0 & 6.6 & 14.6 & 1.6 \\
\hline Grower (soybean meal) & day 9-16 & 14.5 & 10.6 & 25.1 & 2.5 \\
\hline Grower (fishmeal) & day 17 -end & 8.8 & 7.4 & 16.2 & 1.8 \\
\hline
\end{tabular}


Diegem, Belgium). The average of ten measurements per segment per animal was calculated.

\section{Assessment of the impact of FBs on the intestinal microbiota}

At day 15, intestinal content samples of the second half of the different small intestinal segments of all six bird per pen were collected, snap frozen in liquid nitrogen, and subsequently stored at $-80{ }^{\circ} \mathrm{C}$ until further DNA extraction. DNA from intestinal content (duodenum, jejunum and ileum) was extracted using a modified QIAamp DNA Stool mini Kit (Qiagen, Hilden, Germany) protocol. An enzymatic pretreatment with lysozyme and a mechanical disruption step with a bead-beater was added to the original protocol. In brief, frozen intestinal content $(250 \mathrm{mg})$ was transferred into a bead beating tube filled with $0.7 \mathrm{~g}$ of glass beads $(\varnothing 100 \mu \mathrm{m}), 0.6 \mathrm{~g}$ of ceramic beads $(\varnothing$ $1.4 \mathrm{~mm})$ and one glass bead $(\varnothing 3.8 \mathrm{~mm})$. Subsequently, $200 \mu \mathrm{L}$ of TE buffer of $\mathrm{pH} 8(10 \mathrm{mM}$ Tris- $\mathrm{HCl}$ and 1 mM EDTA (Sigma Aldrich, Steinheim, Germany)) and $125 \mu \mathrm{L}$ of freshly prepared lysozyme $(100 \mathrm{mg} / \mathrm{mL}$, Sigma Aldrich) was added. After homogenizing by vortex mixing $(1 \mathrm{~min})$, samples were incubated at $37{ }^{\circ} \mathrm{C}$ for $45 \mathrm{~min}$ at $1000 \mathrm{rpm}$ on a Thermomixer compact shaker incubator (Eppendorf, Hamburg, Germany). The final volume was adjusted to $2 \mathrm{~mL}$ with ASL buffer (Qiagen) and samples were bead beated for $10 \mathrm{~s}$ at $6000 \mathrm{rpm}$ on a Precellys 24-Dual homogenizer (Bertin Technologies, Montigny le Bretonneux, France). Further DNA extraction was performed with the QIAamp DNA Stool mini kit (Qiagen) in accordance with the manufacturer's instructions. DNA integrity was evaluated by loading $3 \mu \mathrm{L}$ of DNA on a $0.8 \%$ agarose gel stained with ethidium bromide. The purity and concentration of the extracted DNA were measured using ultraviolet absorption at 260/280 nm and 230/280 $\mathrm{nm}$ ratio (NanoDrop 1000 spectrophotometer, Thermo Scientific, Waltham, MA, USA).

Denaturing gradient gel electrophoresis (DGGE) separates DNA fragments of the same length but with different base-pair sequences. DNA fragments were generated from the small intestinal content DNA samples applying community PCR with universal bacterial primers targeting the variable V3 region of the $16 \mathrm{~S}$ ribosomal RNA. The nucleotide sequences of the primers were as follows: forward primer F341 with GC clamp 5'-CGC CCG CCG CGC GCG GCG GGC GG GCG GGG GCA CGG GGGG - CCT ACG GGA GGC AGC AG 3' and reverse primer R518 5'ATT ACC GCG GCT GCT GG-3' [35]. PCR amplification was performed in duplicate using a Mastercycler Gradient (Eppendorf, Hamburg, Germany), and each PCR reaction was done in a $45 \mu \mathrm{L}$ total reaction mixture using $3 \mu \mathrm{L}$ of the DNA sample $(4 \mathrm{ng} / \mu \mathrm{L})$, $0.125 \mu \mathrm{M}$ of each of the primers, $100 \mu \mathrm{M}$ deoxynucleotide triphosphate (dNTP) (Peqlab Biotechnologie GmbH,
Erlangen, Germany), and $0.6 \mu \mathrm{L}$ of peqGOLD TaqDNA-Polymerase $(5 \mathrm{U} / \mu \mathrm{L})$ (Peqlab). The PCR conditions used were 1 cycle of $94{ }^{\circ} \mathrm{C}$ for $5 \mathrm{~min}$, followed by 9 cycles of $94{ }^{\circ} \mathrm{C}$ for $30 \mathrm{~s}, 64{ }^{\circ} \mathrm{C}$ for $40 \mathrm{~s}$ (decreased by $0.5^{\circ} \mathrm{C} /$ cycle) and $72{ }^{\circ} \mathrm{C}$ for $40 \mathrm{~s}$. Subsequently 19 cycles of $94{ }^{\circ} \mathrm{C}$ for $30 \mathrm{~s}, 56{ }^{\circ} \mathrm{C}$ for $40 \mathrm{~s}$ and $72{ }^{\circ} \mathrm{C}$ for $40 \mathrm{~s}$, followed by one cycle of $72{ }^{\circ} \mathrm{C}$ for 4 min, were run. DGGE was performed as described by [35] with the INGENYPhorU- $2 \times 2$ system (Ingeny, Goes, The Netherlands). Briefly, amplicons were separated using a 30 to $60 \%$ denaturating gradient [35]. $30 \mu \mathrm{L}$ of the PCR product was loaded and electrophoresis was performed at $100 \mathrm{~V}$ for $16 \mathrm{~h}$ at $60{ }^{\circ} \mathrm{C}$. Each gel included four standard reference lanes containing amplicons of 12 bacterial species for normalization and comparison between gels. DGGE gels were stained with 1x SYBR Green I (Sigma-Aldrich) for 30 min. Fingerprinting profiles were visualized using the Bio Vision Imaging system (Peqlab) and the Vision-Capt software (Vilber Lourmat, Marne-la-Vallée, France). The microbial profiles were processed with GelCompar II vs. 6.6 (Applied Maths NV, Sint-Martens-Latem, Belgium).

The similarity between DGGE-profiles, given as a percentage, was analyzed using the Dice similarity coefficient, derived from presence or absence of bands. On the basis of a distance matrix, which was generated from the similarity values, dendrograms were constructed using the unweighted pair group method with arithmetic means (UPGMA) as clustering-method. The microbial richness $(R)$ was assessed as the number of bands within a profile.

Low-GC-containing operational taxonomic units (OTUs) were selected for identification based on the differences between the DGGE patterns of the control group compared to the FBs contaminated group. After extraction of the selected bands, and reapplication on DGGE to confirm their positions relative to the original sample, the respective 16S-fragments were sequenced (LGC Genomics, Berlin, Germany) and aligned to the NCBI GenBank prokaryotic $16 \mathrm{~S}$ ribosomal RNA database using the standard nucleotide BLASTN 2.2.30+ (nucleotide basic local alignment search tool) [36].

\section{Evaluation of the consequences of FBs exposure on necrotic enteritis Quantification of total C. perfringens by $q P C R$}

Total C. perfringens in ileal content samples, collected before experimental $C$. perfringens challenge (day 15), was quantified using the cpa gene (encoding alpha toxin) as target gene. qPCR was performed using SYBR-green $2 \mathrm{x}$ master mix (Bioline, Brussels, Belgium) in a Bio-Rad CFX-384 system. Each reaction was done in triplicate in a $12 \mu \mathrm{L}$ total reaction mixture using $2 \mu \mathrm{L}$ of the DNA sample and $0.5 \mu \mathrm{M}$ final qPCR primer concentration (Table 2). The qPCR conditions were 1 cycle of $95^{\circ} \mathrm{C}$ for 
Table 2 Primer sequences used for (q)PCR analyses

\begin{tabular}{lllll}
\hline Target & Forward primer & Reverse primer & Analysis & Reference \\
\hline cpa & AGT CTA CGC TTG GGA TGG AA & TाT CCT GGG TTG TTC ATT TC & PCR & [55] \\
cpa & GTT GAT AGC GCA GGA CAT GTT AAG & CAT GTA GTC ATC TGT TCC AGC ATC & qPCR & [56] \\
netB & TGA TAC CGC TTC ACA TAA AGG T & ACC GTC CT AGT CTC AAC AAA T & PCR & [30] \\
netB & TCA ATT GGT TAT TCT ATA GGC GGT A & ATA TGA AGC ATT TAT TCC AGC ACC A & PCR \\
rpoA & ACA TCA TTA GCG TTG TCA GTT AAA G & GAG GTT ATG GAA TAA CTC TTG GTA ATG & [30] \\
rpoA & CCA TCT GTT TTा ATA TCT GCT CCA GTA & GGA AGG TGA AGG ACC AAA AAC TAT T & qPCR & [57] \\
\hline
\end{tabular}

Sequences are presented from $5^{\prime}$ to $3^{\prime}$.

10 min, followed by 40 cycles of $95^{\circ} \mathrm{C}$ for $30 \mathrm{~s}, 60{ }^{\circ} \mathrm{C}$ for $30 \mathrm{~s}$, and stepwise increase in the temperature from $65^{\circ}$ to $95^{\circ} \mathrm{C}$ (at $10 \mathrm{~s} / 0.5^{\circ} \mathrm{C}$ ). Melting curve data were analyzed to confirm the specificity of the reaction. For construction of the standard curve, the PCR product was generated using the standard PCR primers (Table 2) and DNA from $C$. perfringens strain CP56. After purification (MSB Spin PCRapace, Stratec Molecular, Berlin, Germany) and determination of the DNA concentration with a Nanodrop ND 1000 spectrophotometer (Nanodrop Technologies, Wilmingtom, DE, USA), the concentration of the linear dsDNA standard was adjusted to $1 \times 10^{8}$ to $1 \times 10^{1}$ copies per $\mu \mathrm{L}$ with each step differing by 10 fold. The copy numbers of samples (copies/g intestinal content) were determined by reading off the standard series with the $\mathrm{Ct}$ values of the samples.

\section{C. perfringens infection trial}

The remaining 28 animals per pen were used in a $C$. perfringens experimental infection trial as previously described [30]. The BW of all animals was measured on day 16 and day 21. Gumboro vaccine (Nobilis Gumboro D78, MSD Animal Health, Brussels, Belgium) was administered on day 16 in the drinking water of all cages. Both groups were experimentally infected with an oral bolus of $4.10^{8}$ cfu C. perfringens strain 56 on days 17, 18, 19 and 20. On day 21, 22 and 23, each day one third of each group was euthanized by overdose sodium pentobarbital and immediately submitted to necropsy. Macroscopic NE lesion scoring of the small intestines (duodenum, jejunum and ileum) was performed single-blinded as follows; 0 no gross lesions; 1 small focal necrosis or ulceration (one to five foci); 2 focal necrosis or ulceration (six to 15 foci); 3 focal necrosis or ulceration (16 foci or more); 4 patches of necrosis of 2 to $3 \mathrm{~cm}$ long; 5 diffuse necrosis typical field cases, partially adapted from [37]. Chickens with a lesion score of 1 or more were classified as NE positive.

\section{In vitro assessment of the effect of $F B_{1}$ on $C$. perfringens growth, and cpa and net $B$ transcription}

Following concentrations of $\mathrm{FB}_{1}$ were tested for their effect on C. perfringens growth and toxin production: 0 ,
$0.2,2$ and $20 \mu \mathrm{g} \mathrm{FB} / \mathrm{mL}$. All tests were performed in triplicate.

The C. perfringens inoculum was 1:1000 diluted in TGY medium, containing the different concentrations of $\mathrm{FB}_{1}$, and incubated anaerobically at $37{ }^{\circ} \mathrm{C}$. A growth curve was produced by bacterial plating of a ten-fold dilution series of the culture at $0,2,3,4,5,6,7,8$ and 24 $\mathrm{h}$ after inoculation. Ten-fold dilutions were prepared in phosphate buffered saline (PBS), and subsequently plated on Colombia agar with 5\% sheep blood. After anaerobic incubation overnight at $37{ }^{\circ} \mathrm{C}$, the number of colony forming units $(\mathrm{cfu}) / \mathrm{mL}$ was determined.

The impact of $\mathrm{FB}_{1}$ on cpa (alpha toxin) and netB (netB toxin) transcription was tested by qRT-PCR. The C. perfringens inoculum was 1:10 000 diluted in TGY medium, containing the different concentrations of $\mathrm{FB}_{1}$, and incubated anaerobically at $37{ }^{\circ} \mathrm{C}$ until an optical density (OD) of 0.6-1.0 was measured at a wavelength of 600 $\mathrm{nm}$ (6h of incubation). The transcription levels of $c p a$ and netB in the presence of $\mathrm{FB}_{1}$ were compared to non$\mathrm{FB}_{1}$ contaminated test conditions and normalized to the housekeeping gene rpoA, encoding RNA polymerase subunit A. Total RNA was isolated using SV total RNA Isolation system (Promega, Leiden, The Netherlands). RNA was treated with Turbo DNA-free kit (Ambion, Austin, TX, USA) per the manufacturer's instructions to remove genomic DNA contamination. Subsequently, RNA was converted to cDNA with iScript cDNA Synthesis Kit (Bio-Rad, Temse, Belgium). qRT-PCR was performed using SYBR-green 2x master mix (Bioline, Brussels, Belgium) in a Bio-Rad CFX-384 system. Each reaction was done in triplicate in a $12 \mu \mathrm{L}$ total reaction mixture using $2 \mu \mathrm{L}$ of cDNA sample and $0.5 \mu \mathrm{M}$ final qPCR primer concentration (Table 2). The qPCR conditions were as described above for total $C$. perfringens determination in ileal content samples. For construction of the standard curve, the PCR product was generated using the standard PCR primers (Table 2).

\section{Statistical analyses}

Statistical program SPSS version 22 was used for data analysis. To compare the number of NE positive birds (lesion score $\geq 2$ ) between different groups, binomial 
logistic regression was used. All other parameters, including BW relative organ weight, length of small intestines, $\mathrm{Sa} / \mathrm{So}$ ratio, villus height/crypt depth measurements, concentration of $C$. perfringens in ileal digesta, in vitro assessment of clostridial growth, and cpa and netB transcription were analyzed by an independent Student's t-test, after determination of normality. Significance level was set at 0.05 .

\section{Results}

\section{FBs negatively affect sphingolipid metabolism}

The inhibition of ceramide synthase by FBs causes an intracellular accumulation of sphingoid bases, mainly sphinganine. An increased $\mathrm{Sa} / \mathrm{So}$ is suggested to be the most sensitive biomarker to FBs intoxication in many animal species [2]. The plasma $\mathrm{Sa} / \mathrm{So}$ ratio was 1.5 fold higher in animals fed the FBs contaminated diet compared to the control animals, $0.21 \pm 0.016$ versus $0.14 \pm 0.014$, respectively $(P<0.001)$. No significant differences were observed in BW between the control group and the FBs contaminated group (Table 3$)$. A trend $(P=0.060)$ was observed for an increased relative weight of liver in chickens fed the FBs contaminated diet (3.69 $\pm 0.134 \%$ of BW) compared to the control group $(3.39 \pm 0.081 \%$ of $\mathrm{BW})$. Relative weight of bursa, spleen, proventriculus, ventriculus, kidneys, lungs and heart did not differ between both experimental groups (data not shown).

\section{FBs reduce total small intestinal length, ileal villus height and crypth depth}

The total length of the small intestine was significantly $(P=0.033)$ decreased in birds of the FBs contaminated group compared to the control group $(130.5 \pm 2.37$ and $139.0 \pm 2.95 \mathrm{~cm}$, respectively). No differences were observed in the relative percentage of length of the different segments of the small intestine (Table 4).

Feeding a FBs contaminated diet significantly reduced villus height $(P=0.002)$ and crypt depth $(P=0.011)$ in ileum (Table 5). No effect was observed on ileal villus to crypt ratio. No effect was shown in duodenum and jejunum.

\section{FBs affect the ileal microbiota composition}

DGGE fingerprint of DNA samples of duodenal and jejunal content, applying community PCR with universal bacterial primers targeting the variable V3 region of the $16 \mathrm{~S}$ ribosomal DNA, could not show a difference in microbiota composition between chickens fed the control diet or the FBs contaminated diet. In the duodenum, three clades were observed related to the diversity of OTUs, independent of the treatment. One clade showed five samples with a reduced number of bands in the duodenum. Most samples had an average diversity between 10 and 15 OTUs across the medium GC-range. Some samples consisted of 18 to 31 OTUs of the medium and high GCrange (Additional file 1). No difference in number of OTUs between both experimental groups was shown in the jejunum. OTUs were all located in the medium range of GC-content (Additional file 2). Within ileum content samples a difference of the DGGE fingerprint according to the treatment was seen. The majority of the ileum samples of the control group contained OTUs in the lower GC-range which ascribes them to one clade (Figure 1, clade A). Among the FBs group a clade of clearly reduced diversity is formed by eight samples (Figure 1, clade B). Another group of samples of this treatment group show a similar diversity of OTUs of medium GCcontent compared to the control-group, but the low-GCOTUs were absent (Figure 1, clade C). The dendrogram of DGGE profiles of four chickens (bird 20,31, 33 and 34) of the FBs group showed a high similarity with the control group (Figure 1, clade D).

Subsequently, five low-GC-content OTUs from ileal content samples of the control group, which made the difference in their DGGE-patterns compared to the FBs group, were identified at genus level by sequencing. Based on these results the most affected groups were related to the genera Clostridium and Lactobacillus. OTUs 19 and 20 had 99.09-100\% sequence similarity with the type strain of Candidatus Arthromitus, recently renamed as Candidatus Savagella [38]. OTUs 4 and 13 had $100 \%$ sequence similarity with Lactobacillus johnsonii and, OTU 16 was similar to the sequence of an unknown species of the genus Lactobacillus.

\section{FBs increase the susceptibility for C. perfringens induced} necrotic enteritis

Quantification of total C. perfringens in DNA samples of ileal content (day 15 of animal trial) by qPCR using cpa

Table 3 Bodyweight of broiler chickens measured on day 1, 8, 16 and 21

\begin{tabular}{llllll}
\hline & & Day 1 & Day 8 & Day 16 & Day 21 \\
\hline Control diet & & & BW $(\mathrm{g})$ & \\
& 0 & $43 \pm 3(n=52)$ & $161 \pm 22(n=52)$ & $488 \pm 78(n=43)$ & $794 \pm 134(n=43)$ \\
FBs contaminated diet & + & $44 \pm 4(n=50)$ & $153 \pm 21(n=50)$ & $448 \pm 53(n=41)$ & $754 \pm 88(n=41)$ \\
& 0 & $43 \pm 3(n=51)$ & $176 \pm 26(n=51)$ & $519 \pm 65(n=42)$ & $841 \pm 88(n=42)$ \\
& + & $43 \pm 3(n=51)$ & $168 \pm 23(n=51)$ & $476 \pm 58(n=42)$ & $785 \pm 104(n=42)$ \\
\hline
\end{tabular}


Table 4 Length of small intestinal segments

\begin{tabular}{llll}
\hline Organ & Control diet & FBs contaminated diet & $P$ \\
\hline Total length small intestines $^{\mathrm{a}}(\mathrm{cm})$ & $139.0 \pm 2.95$ & $130.5 \pm 2.37$ & $0.033^{*}$ \\
Duodenum (\% of total length) $^{\mathrm{b}}$ & $16.9 \pm 0.40$ & $17.2 \pm 0.35$ & 0.656 \\
Jejunum (\% of total length) $^{\mathrm{b}}$ & $43.1 \pm 0.69$ & $41.7 \pm 0.45$ & 0.118 \\
lleum (\% of total length) $^{\mathrm{b}}$ & $40.0 \pm 0.71$ & $41.1 \pm 0.37$ & 0.184 \\
\hline
\end{tabular}

Animals were randomly divided in two experimental groups, each group consisting of three pens. One group was fed a control diet and one was fed a FBs contaminated diet (18.6 mg FB $+\mathrm{FB}_{2} / \mathrm{kg}$ feed). Six birds $\left(3{ }^{\lambda} / 3+\right.$ ) per pen were euthanized on day 15 and the length of small intestinal segments was recorded. Data presented as mean \pm SEM.

${ }^{a}$ Total length small intestines including all three segments: duodenum, jejunum and ileum; ${ }^{\mathrm{b}} \%$ of total length $=($ length segment $(\mathrm{cm}) /$ total length small intestines $(\mathrm{cm})) \times 100 ;(*)$ significantly different $(P<0.05) /$ trend $(P<0.10)$.

gene (alpha toxin) showed an increased level in chickens fed a FBs contaminated diet compared to a control diet $(7.5 \pm 0.30$ versus $6.3 \pm 0.24 \log 10$ copies/g intestinal content) $(P=0.027)$.

The number of chickens with NE increased from $29.8 \pm 5.46 \%$ of the birds in the control group to $44.9 \pm 2.22 \%$ of broilers which were fed the FBs contaminated diet $(P=0.047)$. No effect was observed on the mean lesion scores of NE positive broiler chickens (Figure 2). No macroscopic coccidiosis lesions were observed.

Results of the $C$. perfringens growth assay showed no influence of different concentrations of $\mathrm{FB}_{1}$ on the bacterial growth curve in vitro (data not shown). qRT-PCR analyses showed no impact of $\mathrm{FB}_{1}$ on transcription of genes encoding alpha (cpa) and netB (netB) toxin. Results for the $c p a$ and $n e t B$ gene transcription were normalized to the rpoA gene. The relative cpa transcription (log copies cpa/log copies rpoA) was $1.21 \pm 0.008$, $1.21 \pm 0.006,1.21 \pm 0.004$, and $1.22 \pm 0.011$ in the

Table 5 Effect of FBs on villus height and crypt depth

\begin{tabular}{|c|c|c|c|}
\hline & Control diet & FBs contaminated diet & $P$ \\
\hline \multicolumn{4}{|l|}{ Mid-duodenum } \\
\hline Villus height ( $\mu \mathrm{m})$ & $1628 \pm 39.4$ & $1549 \pm 44.8$ & 0.225 \\
\hline Crypt depth $(\mu m)$ & $196 \pm 7.1$ & $197 \pm 7.8$ & 0.999 \\
\hline Villus to crypt ratio & $8 \pm 0.3$ & $8 \pm 0.4$ & 0.337 \\
\hline \multicolumn{4}{|l|}{ Mid-jejunum } \\
\hline Villus height ( $\mu m)$ & $842 \pm 32.0$ & $880 \pm 28.6$ & 0.278 \\
\hline Crypt depth ( $\mu \mathrm{m})$ & $173 \pm 6.4$ & $182 \pm 6.5$ & 0.385 \\
\hline Villus to crypt ratio & $5 \pm 0.2$ & $5 \pm 0.2$ & 0.927 \\
\hline \multicolumn{4}{|l|}{ Mid-ileum } \\
\hline Villus height ( $\mu m)$ & $497 \pm 31.7$ & $393 \pm 16.2$ & $0.002^{*}$ \\
\hline Crypt depth ( $\mu m)$ & $155 \pm 4.4$ & $131 \pm 5.2$ & $0.011^{*}$ \\
\hline Villus to crypt ratio & $3 \pm 0.2$ & $3 \pm 0.1$ & 0.349 \\
\hline \multicolumn{4}{|c|}{$\begin{array}{l}\text { Broiler chickens were fed a control diet or a } \mathrm{FBs} \text { contaminated diet }(18.6 \mathrm{mg} \\
\mathrm{FB}_{1}+\mathrm{FB}_{2} / \mathrm{kg} \text { feed) for } 15 \text { days. Samples of different small intestinal segments of } \\
\text { six birds }\left(3{ }^{*} / 3+\text { ) per pen, three pens per group, were collected at day } 15 .\right. \\
\text { Analysis based on mean of } 10 \text { measurements per segment per animal was } \\
\text { calculated; data are presented as weighted mean } \pm \text { SEM. }\left(^{*}\right) \text { significantly } \\
\text { different }(P<0.05) \text {. }\end{array}$} \\
\hline
\end{tabular}

presence of $0,0.2,2$ or $20 \mu \mathrm{g} \mathrm{FB} 1 / \mathrm{mL}$, respectively. The relative net $B$ transcription (log copies netB/log copies $r p o A)$ was $1.03 \pm 0.016,1.05 \pm 0.013,1.04 \pm 0.015$, and $1.04 \pm 0.007$ in the presence of $0,0.2,2$ or $20 \mu \mathrm{g} \mathrm{FB} / \mathrm{mL}$, respectively.

\section{Discussion}

The ingestion of FBs contaminated feed by broiler chickens, at a level of about $20 \mathrm{mg} \mathrm{FB}_{1}+\mathrm{FB}_{2} / \mathrm{kg}$ feed, affects the intestinal microbial homeostasis. Subsequently, these changes possibly predispose the birds to $C$. perfringens induced NE. To our knowledge, this is the first time such an effect has been demonstrated.

FBs negatively affect broiler health, demonstrated by the increased plasma $\mathrm{Sa} / \mathrm{So}$ ratios in broiler chicks fed a FBs contaminated diet. These results suggest that the sphingolipid metabolism was impaired after exposure to levels of FBs approaching the EU maximum guidance levels [31]. FBs inhibit the ceramide synthase enzyme, causing an intracellular accumulation of sphingoid bases, mainly sphinganine. Since the disruption in the sphingolipid metabolism occurs before other indicators of cell injury, the $\mathrm{Sa} / \mathrm{So}$ ratio is suggested to be the most sensitive biomarker to FB intoxication in many animal species $[13,39]$. A similar increase in the $\mathrm{Sa} / \mathrm{So}$ ratio has been demonstrated in serum of broilers fed 80-100 mg FB $1 / \mathrm{kg}$ feed for 3-4 weeks $[14,40,41]$. Furthermore, a linear dosedependent increase in the $\mathrm{Sa} / \mathrm{So}$ ratio has been observed in the liver of broiler chickens fed $20-80 \mathrm{mg} \mathrm{FB} / \mathrm{kg}$ feed for three weeks [40]. In the present study, the relative weight of liver was increased in broilers fed the FBs contaminated diet. A similar effect has already been observed when broiler chickens were fed a FBs contaminated diet containing $100 \mathrm{mg} \mathrm{FB}$ and $20 \mathrm{mg} \mathrm{FB}_{2} / \mathrm{kg}$ feed for 2-4 weeks [14]. In broilers, this effect was not reported in other studies using low levels $\mathrm{FB}_{1}(<100 \mathrm{mg} / \mathrm{kg}$ feed $)$ $[8,40]$. Dietary exposure to FBs has been associated with histopathological degenerative changes in the hepatocytes including mild vacuolar degeneration and bile duct hyperplasia [42].

Consumption of a diet contaminated with FBs for 15 days reduced small intestinal length, ileal villus height 


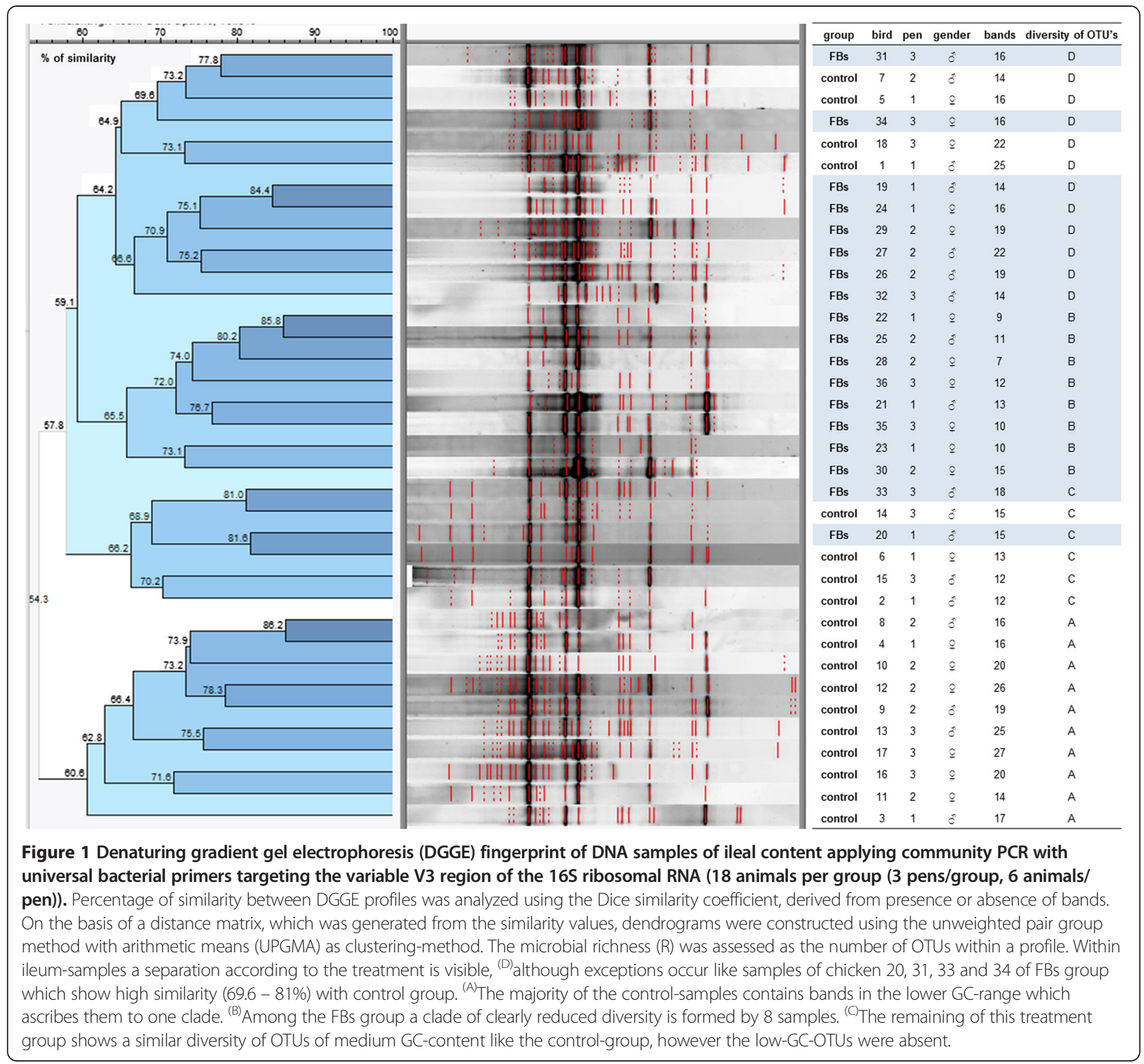

and crypt depth. These results could be related to the negative impact of $\mathrm{FB}_{1}$ on epithelial cell proliferation, reducing villus renewal and impairing intestinal absorption of nutrients [13]. This is in accordance with a previous study, where a decreased villus height was observed in the jejunum of broiler chickens fed high concentrations of FBs (>100 mg FB $1 / \mathrm{kg}$ feed) $[14,15]$. In pigs exposed to FBs for 9 days $(1.5 \mathrm{mg} \mathrm{FB} / \mathrm{kg} \mathrm{BW})$, however, ileal villi tended to be longer [43]. It remains to be determined if this effect on intestinal morphology is induced only by a direct toxic effect of FBs on intestinal epithelial cells, or also indirectly, by the microbiota shift induced by FBs. Longer villi are for example observed in the ileum of chickens treated with $L$. reuteri, indicating that the composition of the intestinal microbiota may indeed affect intestinal morphology [44]. Since the intestinal mucus layer and microbiota are strongly associated, FBs could also modify the microbiota through modulation of the mucus production. Goblet cell hyperplasia was observed in broiler chickens exposed to very high dietary concentration of $\mathrm{FB}_{1}$ (300 $\mathrm{mg} / \mathrm{kg}$ feed) for two weeks [15]. Similarly, it was demonstrated that non-cytotoxic concentrations of DON decreased mucin production in human colonic epithelial goblet cells (HT-29 16E) and porcine intestinal explants [45]. The ingestion of FBs contaminated feed by broiler chickens for 15 days resulted in a modified composition of the intestinal microbiota of the ileum. Based on separation of DNA fragments by electrophoresis of PCR-amplified 16S ribosomal DNA fragments, using polyacrylamide gels containing a linear 


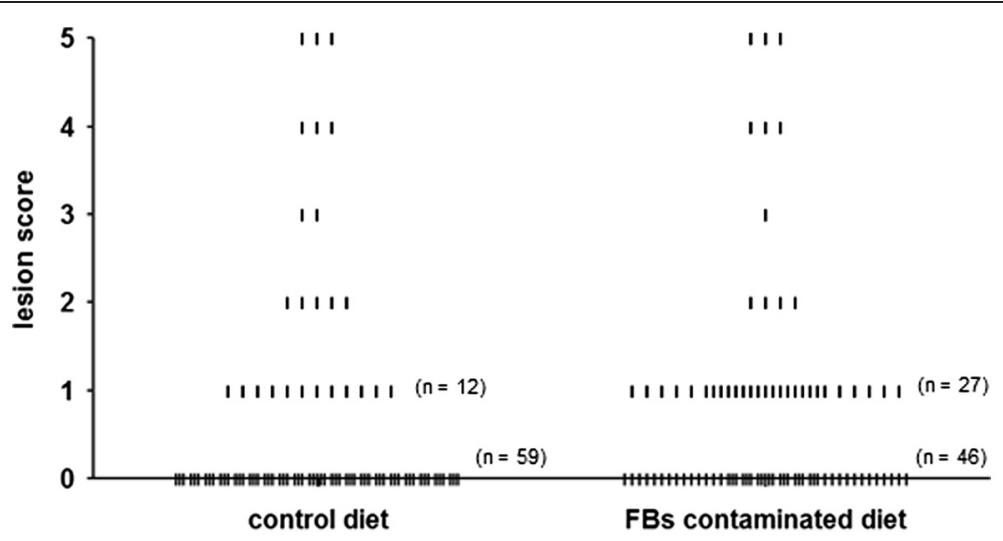

Figure 2 NE lesion score of individual broiler chickens challenged with C. perfringens. Chickens were fed either a control diet or a FBs contaminated diet. Subsequently, birds were orally inoculated with C. perfringens strain 56. Macroscopic intestinal NE lesions in the small intestine (duodenum to ileum) were scored as follow; 0 no gross lesions; 1 small focal necrosis or ulceration (one to five foci); 2 focal necrosis or ulceration (six to $15 \mathrm{foci}$ ); 3 focal necrosis or ulceration (16 or more); 4 patches of necrosis of 2 to $3 \mathrm{~cm}$ long; 5 diffuse necrosis typical field cases. Chickens with NE lesions scores of 1 or more were categorized as NE positive. No effect was observed on the mean lesion scores of NE positive chickens.

gradient of DNA denaturants, the DGGE technique provides a genetic fingerprint of a complex microbial community. The PCR product banding pattern is indicative of the number of bacterial species or assemblages of species that are present [35]. The results clearly indicate a reduced diversity of the ileal microbiota in broiler chickens exposed to FBs compared to the control group. The difference was mainly due to a reduced presence of low-GCcontent OTUs in ileal content samples of FBs exposed animals. Feeding a FBs contaminated diet to broiler chickens was correlated with a decrease in the abundance of Candidatus Arthromitus, recently renamed to Candidatus Savagella [38]. These segmented filamentous bacteria (SFB) are a unique group of uncultivated commensal bacteria within the bacterial family of Clostridiaceae. These SFB are characterized by their attachment to the intestinal epithelium and their important role in modulating host immune systems $[38,46]$. They induce IgA secreting cells and influence the development of the T-cell repertoire $[47,48]$. Stanley et al. [47] demonstrated that the bestknown predisposing factor for necrotic enteritis, coccidiosis, also eliminates or reduces the levels of this immune modulating bacterium. Since coccidiosis and FBs are both predisposing factors for $C$. perfringens induced $\mathrm{NE}$ in broiler chickens, the role of Candidatus Savagella in the pathogenesis of NE needs to be further investigated. It has been suggested that the colonization of the ileum with SFB is correlated with the population of lactobacilli [49]. Lactobacilli belong to the low GC Gram-positive group of Lactobacillales, fermenting sugars to lactic acid [50]. In this study, FBs also modulated the presence of Lactobacillaceae in the ileum. L. johnsonii (OTUs 4 and 13 with sequence similarity of $100 \%$ ) was reduced in FBs exposed birds. L. johnsonii has been extensively investigated for its probiotic activities including pathogen inhibition, epithelial cell-attachment, and immunomodulation [50]. Similar to our results, it was recently demonstrated that $L$. johnsonii was reduced in birds fed fishmeal with or without $C$. perfringens challenge [47,51]. A positive association was demonstrated between crude protein derived from fishmeal and numbers of ileal and caecal $C$. perfringens [52]. L. johnsonii interferes with the colonization and persistence of $C$. perfringens in poultry [53] and some lactobacilli can inhibit growth of $C$. perfringens [54]. It needs to be further investigated whether lactic acid bacteria (LAB) are able to counteract the negative effects of mycotoxins on the intestinal health in poultry.

In conclusion, feeding a FBs contaminated diet at contamination levels approaching the EU maximum guidance level altered the sphingolipid metabolism in broiler chickens without affecting BW gain. FBs modified the composition of the intestinal microbiota of the ileum. DGGE analysis demonstrated a reduced presence of low-GC content OTUs in ileal digesta of birds exposed to FBs, which were subsequently identified as a reduced abundance of Candidatus Savagella and Lactobaccilus spp. such as L. johnsonii. The ileal concentration of total C. perfringens was increased in chickens fed the FBs contaminated diet. Additionally, small intestinal length, ileal villus height, and crypt depth were negatively affected by FBs. The changes in the gut microbiota possibly induced an environment stimulating C. perfringens colonization, and predisposing the birds to necrotic enteritis. The impact of different predisposing factors for NE in broilers, among others coccidiosis, fishmeal and FBs, on intestinal microbiota shows remarkable similarities. The observed predisposing effect is due to the negative impact of FBs on the intestinal microbiota and the animal host, rather than its effect on the bacterium itself. 


\section{Additional files}

Additional file 1: Denaturing gradient gel electrophoresis (DGGE) fingerprint of DNA samples of duodenal content applying community PCR with universal bacterial primers targeting the variable V3 region of the $16 \mathrm{~S}$ ribosomal RNA (18 animals per group (3 pens/group, 6 animals/pen)). Percentage of similarity between DGGE profiles was analyzed using the Dice similarity coefficient, derived from presence or absence of bands. On the basis of a distance matrix, which was generated from the similarity values, dendrograms were constructed using the unweighted pair group method with arithmetic means (UPGMA) as clustering-method. The microbial richness (R) was assessed as the number of OTUs within a profile. Treatment is not reflected by DGGE fingerprint. Independently of treatment three clades are distinguishable concerning the diversity of OTUs: ${ }^{\left({ }^{(A)}\right.}$ one clade with reduced number of bands built by 2 FBs-samples and 3 control-samples, a second clade ${ }^{(B)}$ with average diversity between 10 and 15 OTUs across the medium GC-range and a third clade ${ }^{(C)}$ consisting of 18 to 31 OTUs again in the medium but also high GC-range.

Additional file 2: Denaturing gradient gel electrophoresis (DGGE) fingerprint of DNA samples of jejunal content applying community PCR with universal bacterial primers targeting the variable V3 region of the $16 \mathrm{~S}$ ribosomal RNA (18 animals per group (3 pens/ group, 6 animals/pen)). Percentage of similarity between DGGE profiles was analyzed using the Dice similarity coefficient, derived from presence or absence of bands. On the basis of a distance matrix, which was generated from the similarity values, dendrograms were constructed using the unweighted pair group method with arithmetic means (UPGMA) as clustering-method. The microbial richness (R) was assessed as the number of OTUs within a profile. Treatment is not reflected by DGGE fingerprint. ${ }^{(A)}$ No difference in number of OTUs was demonstrated. In general, all samples OTUs were located in the medium range of GC-content. ${ }^{(1)}$ The banding-pattern of bird 8, control group, is shifted to the lower GC-range what flags it unique in comparison to the others which hardly exhibit bands in this area.

\section{Abbreviations}

BW: Bodyweight; DGGE: Denaturing gradient gel electrophoresis; dNTP: Deoxynucleotide triphosphate; DON: Deoxynivalenol; ELEM: Equin leucoencephalomalacie; $\mathrm{FB}_{1}$ : Fumonisin $\mathrm{B}_{1}$; FBs: Fumonisins; IFN: Interferon; IL: Interleukin; LC-MS/MS: Liquid chromatography-tandem mass spectrometry method; NE: Necrotic enteritis; OD: Optical density; OTUs: Operational taxonomic units; PBS: Phosphate buffered saline; PPE: Porcine pulmonary edema; R: Microbial richness; Sa: Sphinganine; So: Sphingosine; TGY: Tryptone glucose yeast; V:C: Villus-to-crypt ratio; VH: Villus height.

\section{Competing interests}

The authors have the following interests: this work received the financial support from Biomin GmbH, Herzogenburg, Austria. Co-authors Birgit Antlinger and Barbara Novak are employed by Biomin GmbH. There are no patents, products in development or marketed products to declare. This does not alter the authors' adherence to all the VETERINARY RESEARCH policies on sharing data and materials.

\section{Authors' contributions}

Conceived the study, participated in its design and coordination GA, SC, FP, RD, FH, AM, FVI. Performed the experiments: GA, VE, MDV, MV, BA, BN. Analyzed the data: GA, SC, FP, RD, VE, BA, AM, FVI. Contributed reagents/ materials/analysis tools: RD, VE, ME, SDS, BA, BN. Contributed to writing of the manuscript: GA, SC, FP, RD, FH, AM, FVI. All authors read and approved the final manuscript.

\section{Acknowledgements}

The technical assistance of Muyle J., Detavernier C., Puttevils C., Ameye D., Van Hecke M., De Bock Y. was gratefully appreciated. Delezie E. from the Institute for Agricultural and Fisheries Research (Melle, Belgium) is acknowledged for formulation of the feed. The authors gratefully appreciated the excellent assistance of many PhD students from the Department of Pharmacology, Toxicology and Biochemistry and the Department of Bacteriology, Pathology and Avian Diseases.

\section{Author details}

'Department of Pathology, Bacteriology and Avian Diseases, Faculty of Veterinary Medicine, Ghent University, Salisburylaan 133, 9820 Merelbeke, Belgium. ${ }^{2}$ Department of Pharmacology, Toxicology and Biochemistry, Faculty of Veterinary Medicine, Ghent University, Salisburylaan 133, 9820 Merelbeke, Belgium. ${ }^{3}$ Department of Applied Biosciences, Faculty of Bioscience Engineering, Ghent University, Valentin Vaerwyckweg 1, 9000 Ghent, Belgium. ${ }^{4}$ Department of Bio-analysis, Faculty of Pharmaceutical Sciences, Ghent University, Ottergemsesteenweg 460, 9000 Ghent, Belgium. ${ }^{5}$ Biomin Research Center, Technopark 1, 3430 Tulln, Austria.

Received: 20 May 2015 Accepted: 28 July 2015

Published online: 23 September 2015

\section{References}

1. Binder EM (2007) Managing the risk of mycotoxins in modern feed production. Anim Feed Sci Tech 133:149-166

2. Voss KA, Smith GW, Haschek WM (2007) Fumonisins: Toxicokinetics, mechanism of action and toxicity. Anim Feed Sci Tech 137:299-325

3. Streit E, Naehrer K, Rodrigues I, Schatzmayr G (2013) Mycotoxin occurrence in feed and feed raw materials worldwide: long term analysis with special focus on Europe and Asia. J Sci Food Agr 93:2892-2899

4. Schatzmayr G, Streit E (2013) Global occurrence of mycotoxins in the food and feed chain: facts and figures. World Mycotoxin J 6:213-222

5. Wu F (2007) Measuring the economic impacts of Fusarium toxins in animal feeds. Anim Feed Sci Tech 137:363-374

6. Riley RT, Enongene E, Voss KA, Norred WP, Meredith FI, Sharma RP, Spitsbergen J, Williams DE, Carlson DB, Merrill AH (2001) Sphingolipid perturbations as mechanisms for fumonisin carcinogenesis. Environ Health Perspect 109:301-308

7. Ledoux DR, Bermudez AJ, Rottinghaus GE (1996) Effects of feeding Fusarium moniliforme culture material, containing known levels of fumonisin $B_{1}$, in the young turkey poult. Poultry Sci 75:1472-1478

8. Weibking TS, Ledoux DR, Bermudez AJ, Turk JR, Rottinghaus GE, Wang E, Merrill AH (1993) Effects of feeding Fusarium moniliforme culture material, containing known levels of fumonisin $B_{1}$, on the young broiler chick. Poultry Sci 72:456-466

9. Tardieu D, Bailly JD, Benard G, Tran TS, Guerre P (2004) Toxicity of maize containing known levels of fumonisin $B_{1}$ during force-feeding of ducks. Poultry Sci 83:1287-1293

10. Tran ST, Auvergne A, Benard G, Bailly JD, Tardieu D, Babile R, Guerre P (2005) Chronic effects of fumonisin $B_{1}$ on ducks. Poultry Sci 84:22-28

11. Kubena LF, Harvey RB, Buckley SA, Bailey RH, Rottinghaus GE (1999) Effects of long-term feeding of diets containing moniliformin, supplied by Fusarium fujikuroi culture material, and fumonisin, supplied by Fusarium moniliforme culture material, to laying hens. Poultry Sci 78:1499-1505

12. Antonissen $G$, Devreese $M$, Van Immerseel F, De Baere $S$, Hessenberger $S$, Martel A, Croubels S (2015) Chronic exposure to deoxynivalenol has no influence on the oral bioavailability of fumonisin $B_{1}$ in broiler chickens. Toxins 7:560-571

13. Bouhet S, Oswald IP (2007) The intestine as a possible target for fumonisin toxicity. Mol Nutr Food Res 51:925-931

14. Rauber RH, Oliveira MS, Mallmann AO, Dilkin P, Mallmann CA, Giacomini LZ, Nascimento VP (2013) Effects of fumonisin $B_{1}$ on selected biological responses and performance of broiler chickens. Pesqui Vet Brasil 33:1081-1086

15. Brown TP, Rottinghaus GE, Williams ME (1992) Fumonisin mycotoxicosis in broilers: performance and pathology. Avian Dis 36:450-454

16. Bouhet S, Le Dorze E, Peres S, Fairbrother JM, Oswald IP (2006) Mycotoxin fumonisin $B_{1}$ selectively down-regulates the basal IL-8 expression in pig intestine: in vivo and in vitro studies. Food Chem Toxicol 44:1768-1773

17. Bracarense AP, Lucioli J, Grenier B, Drociunas Pacheco G, Moll W-D, Schatzmayr G, Oswald IP (2012) Chronic ingestion of deoxynivalenol and fumonisin, alone or in interaction, induces morphological and immunological changes in the intestine of piglets. Br J Nutr 107:1776-1786

18. Basso K, Gomes F, Bracarense AP (2013) Deoxynivanelol and fumonisin, alone or in combination, induce changes on intestinal junction complexes and in E-cadherin expression. Toxins 5:2341-2352

19. Devriendt B, Verdonck F, Wache Y, Bimczok D, Oswald IP, Goddeeris BM, Cox E (2009) The food contaminant fumonisin $B_{1}$ reduces the maturation of porcine CD11R1+ intestinal antigen presenting cells and antigen-specific 
immune responses, leading to a prolonged intestinal ETEC infection. Vet Res 40:40

20. Oswald IP, Desautels C, Laffitte J, Fournout S, Peres SY, Odin M, Le Bars P, Le Bars J, Fairbrother JM (2003) Mycotoxin fumonisin $B_{1}$ increases intestinal colonization by pathogenic Escherichia coli in pigs. Appl Environ Microb 69:5870-5874

21. Grenier B, Bracarense AP, Schwartz HE, Trumel C, Cossalter AM, Schatzmayr G, Kolf-Clauw M, Moll WD, Oswald IP (2012) The low intestinal and hepatic toxicity of hydrolyzed fumonisin $B_{1}$ correlates with its inability to alter the metabolism of sphingolipids. Biochem Pharmacol 83:1465-1473

22. Burel C, Tanguy M, Guerre P, Boilletot E, Cariolet R, Queguiner M, Postollec G, Pinton P, Salvat G, Oswald IP (2013) Effect of low dose of fumonisins on pig health: immune status, intestinal microbiota and sensitivity to salmonella. Toxins 5:841-864

23. Songer JG (1996) Clostridial enteric diseases of domestic animals. Clin Microbiol Rev 9:216-234

24. Timbermont L, Haesebrouck F, Ducatelle R, Van Immerseel F (2011) Necrotic enteritis in broilers: an updated review on the pathogenesis. Avian Pathol 40:341-347

25. Keyburn AL, Boyce JD, Vaz P, Bannam TL, Ford ME, Parker D, Di Rubbo A, Rood JI, Moore RJ (2008) NetB, a new toxin that is associated with avian necrotic enteritis caused by Clostridium perfringens. PLoS Pathog 4:e26

26. Van Immerseel F, De Buck J, Pasmans F, Huyghebaert G, Haesebrouck F, Ducatelle R (2004) Clostridium perfringens in poultry: an emerging threat for animal and public health. Avian Pathol 33:537-549

27. Van Immerseel F, Rood Jl, Moore RJ, Titball RW (2009) Rethinking our understanding of the pathogenesis of necrotic enteritis in chickens. Trends Microbiol 17:32-36

28. Shojadoost B, Vince AR, Prescott JF (2012) The successful experimental induction of necrotic enteritis in chickens by Clostridium perfringens: a critical review. Vet Res 43:74

29. Williams R (2005) Intercurrent coccidiosis and necrotic enteritis of chickens: rational, integrated disease management by maintenance of gut integrity. Avian Pathol 34:159-180

30. Antonissen G, Van Immerseel F, Pasmans F, Ducatelle R, Haesebrouck F, Timbermont L, Verlinden M, Janssens GPJ, Eeckhaut V, Eeckhout M, De Saeger S, Hessenberger S, Martel A, Croubels S (2014) The mycotoxin deoxynivalenol predisposes for the development of Clostridium perfringens-induced necrotic enteritis in broiler chickens. PLoS One 9:e108775

31. European Commission (2006) Commission recommendation of 17 August 2006 on the presence of deoxynivalenol, zearalenone, ochratoxin A, T-2 and HT-2 and fumonisins in products intended for animal feeding. Off J Eur Union L229:7-9

32. Leslie JF, Plattner RD, Desjardins AE, Klittich CJR (1992) Fumonisin $B_{1}$ production by strains from different mating populations of Gibberella fujikuroi (Fusarium section liseola). Phytopathology 82:341-345

33. Gholamiandehkordi AR, Timbermont $L$, Lanckriet A, Van Den Broeck W, Pedersen K, Dewulf J, Pasmans F, Haesebrouck F, Ducatelle R, Van Immerseel F (2007) Quantification of gut lesions in a subclinical necrotic enteritis model. Avian Pathol 36:375-382

34. Monbaliu S, Van Poucke C, Detavernier C, Dumoulin F, Van De Velde M, Schoeters E, Van Dyck S, Averkieva O, Van Peteghem C, De Saeger S (2009) Occurrence of mycotoxins in feed as analyzed by a multi-mycotoxin LC-MS/MS method. J Agr Food Chem 58:66-71

35. Muyzer G, de Waal EC, Uitterlinden AG (1993) Profiling of complex microbial-populations by denaturing gradient gel-electrophoresis analysis of polymerase chain reaction-amplified genes coding for $16 \mathrm{~s}$ rRNA. Appl Environ Microbiol 59:695-700

36. Zhang Z, Schwartz S, Wagner L, Miller W (2000) A greedy algorithm for aligning DNA sequences. J Comput Biol 7:203-214

37. Keyburn AL, Sheedy SA, Ford ME, Williamson MM, Awad MM, Rood Jl, Moore RJ (2006) Alpha-toxin of Clostridium perfringens is not an essential virulence factor in necrotic enteritis in chickens. Infect Immun 74:6496-6500

38. Thompson CL, Mikaelyan A, Brune A (2013) Immune-modulating gut symbionts are not "Candidatus Arthromitus". Mucosal Immunol 6:200-201

39. Riley RT, Hinton DM, Chamberlain WJ, Bacon CW, Wang E, Merrill AH, Voss KA (1994) Dietary fumonisin $B_{1}$ Induces disruption of sphingolipid metabolism in Sprague-Dawley rats - a new mechanism of nephrotoxicity. J Nutr 124:594-603

40. Henry MH, Wyatt RD, Fletcher OJ (2000) The toxicity of purified fumonisin $B_{1}$ in broiler chicks. Poultry Sci 79:1378-1384
41. Poersch AB, Trombetta F, Braga ACM, Boeira SP, Oliveira MS, Dilkin P, Mallmann CA, Fighera MR, Royes LFF, Oliveira MS, Furian AF (2014) Involvement of oxidative stress in subacute toxicity induced by fumonisin $\mathrm{B}_{1}$ in broiler chicks. Vet Microbiol 174:180-185

42. Tessari ENC, Oliveira CAF, Cardoso ALSP, Ledoux DR, Rottinghaus GE (2006) Effects of aflatoxin B-1 and fumonisin $B_{1}$ on body weight, antibody titres and histology of broiler chicks. Brit Poultry Sci 47:357-364

43. Lessard M, Boudry G, Seve B, Oswald IP, Lalles JP (2009) Intestinal physiology and peptidase activity in male pigs are modulated by consumption of corn culture extracts containing fumonisins. J Nutr 139:1303-1307

44. Dunham HJ, Laster SM, Edens FW, Casas IA, Dobrogosz WJ (1993) Immunomodulation of stressor-associated diseases in neonate chickens and turkeys by gastrointestinal colonization with Lactobacillus reuteri. J Immunol 150:A197-A197

45. Pinton P, Graziani F, Pujol A, Nicoletti C, Paris O, Ernouf P, Pasquale ED, Perrier J, Oswald IP, Maresca M (2015) Deoxynivalenol inhibits the expression by goblet cells of intestinal mucins through a PKR and MAP kinase-dependent repression of the resistin-like molecule beta. Mol Nutr Food Res 59:1076-1087

46. Snel J, Heinen PP, Blok HJ, Carman RJ, Duncan AJ, Allen PC, Collins MD (1995) Comparison of 165 rRNA sequences of segmented filamentous bacteria isolated from mice, rats, and chickens and proposal of "Candidatus Arthromitus". Int J Syst Bacteriol 45:780-782

47. Stanley D, Wu SB, Rodgers N, Swick RA, Moore RJ (2014) Differential responses of cecal microbiota to fishmeal, Eimeria and Clostridium perfringens in a necrotic enteritis challenge model in chickens. PLoS One 9:e104739

48. Ericsson AC, Hagan CE, Davis DJ, Franklin CL (2014) Segmented filamentous bacteria: commensal microbes with potential effects on research. Comp Med 64:90-98

49. Liao N, Yin Y, Sun G, Xiang C, Liu D, Yu HD, Wang X (2012) Colonization and distribution of segmented filamentous bacteria (SFB) in chicken gastrointestinal tract and their relationship with host immunity. FEMS Microbiol Ecol 81:395-406

50. Pridmore RD, Berger B, Desiere F, Vilanova D, Barretto C, Pittet AC, Zwahlen MC, Rouvet M, Altermann E, Barrangou R, Mollet B, Mercenier A, Klaenhammer T, Arigoni F, Schell MA (2004) The genome sequence of the probiotic intestinal bacterium Lactobacillus johnsonii NCC 533. Proc Natl Acad Sci U S A 101:2512-2517

51. Wu SB, Stanley D, Rodgers N, Swick RA, Moore RJ (2014) Two necrotic enteritis predisposing factors, dietary fishmeal and Eimeria infection, induce large changes in the caecal microbiota of broiler chickens. Vet Microbiol 169:188-197

52. Drew M, Syed N, Goldade B, Laarveld B, Van Kessel A (2004) Effects of dietary protein source and level on intestinal populations of Clostridium perfringens in broiler chickens. Poultry Sci 83:414-420

53. La Ragione RM, Narbad A, Gasson MJ, Woodward MJ (2004) In vivo characterization of Lactobacillus johnsonii Fl9785 for use as a defined competitive exclusion agent against bacterial pathogens in poultry. Lett Appl Microbiol 38:197-205

54. Dec M, Puchalski A, Urban-Chmiel R, Wernicki A (2014) Screening of Lactobacillus strains of domestic goose origin against bacterial poultry pathogens for use as probiotics. Poultry Sci 93:2464-2472

55. Baums CG, Schotte U, Amtsberg G, Goethe R (2004) Diagnostic multiplex PCR for toxin genotyping of Clostridium perfringens isolates. Vet Microbiol 100:11-16

56. Gholamiandekhordi AR, Ducatelle R, Heyndrickx M, Haesebrouck F, Van Immerseel F (2006) Molecular and phenotypical characterization of Clostridium perfringens isolates from poultry flocks with different disease status. Vet Microbiol 113:143-152

57. Cheung JK, Keyburn AL, Carter GP, Lanckriet AL, Van Immerseel F, Moore RJ, Rood Jl (2010) The VirSR two-component signal transduction system regulates NetB toxin production in Clostridium perfringens. Infect Immun 78:3064-3072 